\title{
Analyse et méthodes numériques pour la simulation de phénomènes d'interaction fluide-structure
}

\author{
Céline Grandmont* \& Yvon Maday ${ }^{\dagger}$
}

\begin{abstract}
Résumé
Dans cet article, nous présentons une synthèse d'un certain nombre de résultats sur l'analyse mathématique et numérique de phénomènes d'interaction entre un fluide et un solide mobile: rigide ou déformable. Les applications où de telles interactions se produisent sont nombreuses, en aérodynamique, en hydrodynamique, en biomécanique... Nous pourrions citer beaucoup d'exemples: écoulement autour d'un bateau, écoulement sanguins dans les artères ${ }^{1}$, pour l'hydrodynamique (fluide en phase liquide), écoulement autour d'ailes d'avion, étude de l'influence des vents sur le tablier d'un pont, pour l'aéroélasticité ${ }^{2}$ (fluide en phase gazeuse)... Tous ces phénomènes sont dits couplés car le comportement du fluide dépend de celui de la structure et réciproquement.
\end{abstract}

\section{Introduction}

Nous nous plaçons ici dans le cadre le plus général où la déformation de la structure est telle qu'il en résulte une déformation non négligeable du domaine occupé par le fluide. L'interaction se produit à plusieurs niveaux. Tout d'abord le déplacement de la structure modifie la forme du domaine fluide, ce déplacement de la structure résulte lui même d'une action des forces extérieures mais aussi des efforts du fluide sur la frontière du solide. Il est clair à partir de ces quelques explications que le phénomène en présence est très non linéaire et requiert une analyse minutieuse. De nombreuses études ont été faites sur ce thème, mais essentiellement dans le cadre de petites déformations de la structure (vibrations). Le domaine fluide est alors considéré comme fixe. On pourra consulter le livre [26], dans cette voie.

\section{Formulation mathématique du problème}

L'écoulement est supposé visqueux et incompressible. Nous placerons donc cette étude dans le cadre où le fluide est modélisé par les équations de Navier-Stokes incompressibles. Ce fluide interagit avec un solide que nous supposons, dans un premier temps, régi par des équations linéaires. Afin de récupérer le savoir faire accumulé sur la résolution de ce type d'équations, on prévoit d'utiliser le formalisme classique des modèles régissant le fluide et la structure: formulation lagrangienne pour la structure et eulérienne pour le fluide. Ce cadre permet, en effet, de traiter de nombreuses applications, en particulier, en modifiant les lois

"Laboratoire d'Analyse Numérique, Université Pierre et Marie Curie, 4, place Jussieu, 75252 Paris Cedex 05, France.

†Laboratoire d'Analyse Numérique, Université Pierre et Marie Curie, 4, place Jussieu, 75252 Paris Cedex 05, France, Laboratoire ASCI, Bat. 506, Université Paris Sud, 91405 Orsay, Cedex, France et ONERA, 29 av Division Leclerc, 92322 Châtillon, France.

1. on pourra consulter le site www.crs4.it

2. on pourra consulter le site www.science.gmu./edu/〜rlohner/pages/rlohner.html 
de comportement des milieux en présence. La formulation lagrangienne des équations fait intervenir la configuration de référence de la structure, appelée $\hat{\Omega}_{S}$. Ce peut être l'état initial de la structure, mais ce n'est pas obligatoire. Le comportement de la structure est donc décrit par le déplacement au cours du temps de chaque point par rapport à la configuration de référence. Ainsi, tout point $\mathbf{x}$ de la configuration de référence $\hat{\Omega}_{S}$ occupe, à l'instant $t$ la position $\mathbf{x}(t)=\mathbf{x}+\mathbf{d}(\mathbf{x}, t)$ où $\mathbf{d}$ (déplacement de la structure) satisfait aux équations constitutives de la structure. Pour ce qui est du fluide, la plupart des représentations sont écrites en formulation eulérienne. Ainsi, les inconnues (vitesse, pression,...) sont évaluées à chaque instant et en chaque point du domaine physique. En particulier, dans la situation qui nous intéresse ici, le domaine fluide est délimité, pour une partie au moins de sa frontière, par une structure mobile et donc, varie au cours du temps. On note $\Omega_{F}(t)$ ce domaine au temps $t$. La résolution de la partie fluide consiste donc à : trouver $(\mathbf{u}, p)$ défini sur $\Omega_{F}(t)$ tels que

$$
\begin{aligned}
\frac{\partial \mathbf{u}}{\partial t}-\nu \Delta \mathbf{u}+\mathbf{u} \cdot \nabla \mathbf{u}+\nabla p & =\mathbf{f} & \operatorname{dans} \Omega_{F}(t) \\
\operatorname{div} \mathbf{u} & =0 & \operatorname{dans} \Omega_{F}(t),
\end{aligned}
$$

où u désigne la vitesse du fluide, $p$ sa pression, $\nu$ sa viscosité et $\mathbf{f}$ une force extérieure donnée. Ces équations sont complétées par une donnée initiale

$$
\mathbf{u}(t=0, .)=\mathbf{u}_{0}(.) \quad \text { dans } \Omega_{F}(0)
$$

et des conditions aux limites. Ces conditions aux limites sont de deux types, correspondant à deux types de frontière $\partial \Omega_{F}(t)$ du domaine $\Omega_{F}(t)$. Il y a tout d'abord la partie $\gamma(t)$ où le fluide et la structure se touchent, il y a ensuite la partie $\Gamma_{F}=\partial \Omega_{F}(t) \backslash \gamma(t)$ qui est supposée indépendante du temps et sur laquelle on impose des conditions classiques (c'est-à-dire des conditions de Dirichlet, de Neumann ou autres). On va supposer ici pour simplifier qu'il s'agit de conditions de Dirichlet homogènes et donc

$$
\mathbf{u}(t, .)=\mathbf{0} \quad \operatorname{sur} \Gamma_{F} .
$$

Nous détaillerons ultérieurement les conditions de couplage à l'interface entre le fluide et la structure. Avant cela, nous allons décrire deux exemples d'équations de structure. Pour ce qui est de la structure, plusieurs situations sont envisageables : la structure et le fluide peuvent avoir la même "dimension" ou être modélisés par des équations posées dans des domaines de dimensions différentes. En effet, lorsque le corps tridimensionnel possède une petite épaisseur ou que sa section est faible, des modèles 2D (plaques, coques) ou 1D (poutres, cables) sont classiquement utilisés pour décrire son comportement. En général, il s'agit d'équations posées sur la surface moyenne de l'objet et dont la solution est le déplacement (ou la rotation de la normale) des points de la surface moyenne. Les déplacements des points situés dans l'épaisseur peuvent s'en déduire.

- Nous commençons par donner les équations de l'élasticité linéarisée tridimensionnelle. Nous supposerons que la loi de comportement du matériau est la loi de Hooke (matériau isotrope) et nous appellerons $\lambda$ et $\mu$ les coefficients de Lamé du matériau et $\mathbf{d}$ son déplacement. On note $\mathrm{g}$ la force volumique appliquée à la structure. Les équations s'écrivent alors: pour l'inconnue d définie sur $\hat{\Omega}_{S}$

$$
\frac{\partial^{2} \mathbf{d}}{\partial t^{2}}-\operatorname{div} \sigma(\mathbf{d})=\mathrm{g} \quad \operatorname{dans} \hat{\Omega}_{S}
$$

où le tenseur des contraintes $\sigma$ est donné par

$$
\sigma(\mathbf{d})=\lambda \operatorname{tr} \varepsilon(\mathbf{d}) \mathbf{I} \mathbf{d}+2 \mu \varepsilon(\mathbf{d}),
$$


et où le tenseur $\varepsilon(\mathbf{d})$ représentant le tenseur linéarisé des déformations, s'écrit

$$
\varepsilon(\mathbf{d})=\frac{1}{2}\left(\nabla \mathbf{d}+\nabla \mathbf{d}^{T}\right)
$$

Il faut, bien sûr, rajouter des conditions initiales sur le déplacement et la vitesse, soit par exemple

$$
\mathbf{d}(t=0)=0, \quad \frac{\partial \mathbf{d}}{\partial t}(t=0)=\mathbf{v}_{0} .
$$

Il faut aussi imposer des conditions aux limites sur le bord $\Gamma_{S}$ de $\hat{\Omega}_{S}$ qui n'est pas baigné par le fluide. Nous imposerons ici encore des déplacements nuls pour simplifier la présentation.

Finalement, il nous reste à exprimer le principal, c'est à dire les conditions de raccord, qui traduisent l'interaction entre le fluide et la structure. En définissant $\gamma$ comme la portion de frontière de $\hat{\Omega}_{S}$ en contact avec le fluide, on écrit tout d'abord que $\gamma$ et $\gamma(t)$ sont la même entité (l'une exprimée en lagrangien et l'autre en eulérien).

$$
\forall t, \forall \mathbf{x} \in \gamma, \quad \mathbf{x}+\mathbf{d}(t, \mathbf{x}) \in \gamma(t)
$$

et

$$
\forall t, \forall \mathbf{y} \in \gamma(t), \exists \mathbf{x} \in \gamma, \quad \mathbf{x}+\mathbf{d}(t, \mathbf{x})=\mathbf{y}
$$

On impose donc ensuite que

- sur $\gamma(t)$ le fluide "colle" à la paroi et donc la vitesse du fluide est égale à la vitesse de la structure en des points coïncidants.

$$
\forall t, \forall \mathbf{x} \in \gamma, \quad \mathbf{u}(t, \mathbf{x}+\mathbf{d}(t, \mathbf{x}))=\frac{\partial \mathbf{d}}{\partial t}(t, \mathbf{x})
$$

Nous avons supposé que le fluide était visqueux, dans le cas d'un fluide non visqueux, nous n'aurions que la continuité de la composante normale de la vitesse à l'interface.

- par ailleurs, suivant la loi de l'action et de la réaction, les contraintes normales du fluide sur la structure coïncident avec celles de la structure sur le fluide.

$$
\forall t, \forall \mathbf{x} \in \gamma, \quad T_{F}(\mathbf{u}, p)(t, \mathbf{x})=\sigma(\mathbf{d}) \cdot \mathbf{n}(t, \mathbf{x})
$$

où $T_{F}(\mathbf{u}, p)$ représente les contraintes fluides réécrites sur la configuration de référence.

- Si maintenant, en dimension 3 d'espace, le solide considéré possède une dimension "petite" (épaisseur ou section) par rapport aux autres, on réfêre alors, le plus souvent, à des équations de plaques ou de coques. Les conditions de couplage traduisent encore l'égalité des vitesses ainsi que le fait que les forces exercées sur la structure viennent de la pression et de la viscosité du fluide. Considérons, par exemple, une plaque d'épaisseur $2 e$ et de surface moyenne $\omega$. On suppose que le matériau est linéaire élastique homogène et isotrope. On appelle $E$ son module d'Young, $\underline{\nu}$ son coefficient de Poisson (ces deux coefficients peuvent s'exprimer en fonction des coefficients de Lamé du solide élastique), et $\rho$ sa masse volumique. Avec les notations précédentes on a:

$$
\left.\hat{\Omega}_{s}=\omega \times\right]-e, e[
$$

Nous n'allons présenter, ici, que les équations régissant le comportement du déplacement transverse $d_{3}=d_{3}\left(x_{1}, x_{2}\right)$ (les effets membranaires et en flexion sont, en effet, découplés). 
En formulation forte les équations s'écrivent, en utilisant la convention de sommation sur l'indice répété, et en considérant que les indices grecs varient dans $\{1,2\}$ :

$$
\begin{aligned}
2 \rho e \frac{\partial^{2} d_{3}}{\partial t^{2}}+\frac{2 E e^{3}}{3\left(1-\underline{\nu}^{2}\right)} \Delta^{2} d_{3}= & g_{3}^{+}+g_{3}^{-}+\int_{-e}^{e} f_{3} \\
& +e \frac{\partial\left(g_{\alpha}^{+}-g_{\alpha}^{-}\right)}{\partial x_{\alpha}}+\int_{-e}^{e} x_{3} \frac{\partial f_{3}}{\partial x_{\alpha}}, \text { dans } \omega,
\end{aligned}
$$

où $\mathbf{f}$ désigne une force volumique donnée et $\mathrm{g}^{+}, \mathrm{g}^{-}$des forces surfaciques appliquées respectivement sur $\omega \times\{e\}$ et $\omega \times\{-e\}$. On suppose, d'autre part, que la plaque est encastrée sur son bord latéral: $\partial \omega \times[-e,+e]$. Le déplacement longitudinal est donné par:

$$
d_{\alpha}\left(x_{1}, x_{2}, x_{3}\right)=-x_{3} \frac{\partial d_{3}}{\partial x_{\alpha}}\left(x_{1}, x_{2}\right) .
$$

Nous souhaitons maintenant exprimer les conditions de couplage entre cette plaque en flexion et le fluide visqueux. Si l'on considère que $\gamma=\omega \times\{-e\}$, on a pour tout $\mathbf{x} \in \gamma$, c'est à dire pour tout $\mathbf{x}=\left(x_{1}, x_{2},-e\right)$

$$
\begin{aligned}
& u_{3}(\mathbf{x}+\mathbf{d}(t, \mathbf{x}))=\frac{\partial d_{3}}{\partial t}\left(t, x_{1}, x_{2}\right), \\
& u_{\alpha}(\mathbf{x}+\mathbf{d}(t, \mathbf{x}))=e \frac{\partial^{2} d_{3}}{\partial t \partial x_{\alpha}}\left(t, x_{1}, x_{2}\right) .
\end{aligned}
$$

D'autre part, le fluide exerce sur la plaque des efforts surfaciques. Nous allons donc voir apparaître, dans le second membre des équations (12), les contraintes $\left.T_{F}(\mathbf{u}, p)\right|_{\gamma}=\mathrm{g}^{-}$.

Pour plus de détails sur les équations de plaques et de coques on pourra consulter : [11], [12], [6].

\subsection{Estimations d'énergie a priori}

Supposons que la solution du problème couplé existe et soit suffisamment régulière. Dans ce cas, comme pour beaucoup de systèmes dynamiques mécaniques, il est intéressant d'avoir des notions de conservation d'énergie. On multiplie pour ce faire les équations (1) par u et on intègre sur le domaine $\Omega_{F}(t)$, il vient

$$
\int_{\Omega_{F}(t)} \frac{\partial \mathbf{u}}{\partial t} \mathbf{u}-\nu \int_{\Omega_{F}(t)} \mathbf{u} \Delta \mathbf{u}+\int_{\Omega_{F}(t)}(\mathbf{u} \cdot \nabla) \mathbf{u} \cdot \mathbf{u}+\int_{\Omega_{F}(t)} \nabla p \mathbf{u}=\int_{\Omega_{F}(t)} \mathbf{f u .} .
$$

Après intégration par parties et en tenant compte de (2), on obtient

$$
\int_{\Omega_{F}(t)} \frac{1}{2} \frac{\partial \mathbf{u}^{2}}{\partial t}+\nu \int_{\Omega_{F}(t)}(\nabla \mathbf{u})^{2}+\int_{\Omega_{F}(t)} \mathbf{u} \cdot \nabla \mathbf{u} \cdot \mathbf{u}+\int_{\gamma(t)}\left(p \mathbf{n}-\nu \frac{\partial \mathbf{u}}{\partial \mathbf{n}}\right) \mathbf{u}=\int_{\Omega_{F}(t)} \mathbf{f u} .
$$

On rappelle la formule de Reynolds

$$
\frac{d}{d t} \int_{\Omega_{F}(t)} \phi(x, t) d x=\int_{\Omega_{F}(t)} \frac{\partial \phi(x, t)}{\partial t} d x+\int_{\gamma(t)} \phi \mathbf{w} . \mathbf{n},
$$

où $\mathbf{n}$ est la normale extérieure à $\Omega_{F}(t)$ et $\mathbf{w}$ est la vitesse de chaque point de la frontière $\gamma(t)$. Nous allons l'utiliser avec $\phi=\frac{\mathbf{u}^{2}}{2}$. Par ailleurs, en utilisant la conservation de la masse (l'équation (2)) le terme de convection devient, après intégration par parties

$$
\int_{\Omega_{F}(t)}(\mathbf{u} . \nabla) \mathbf{u . u}=\frac{1}{2} \int_{\gamma(t)} \mathbf{u}^{2} \mathbf{u} . \mathbf{n} .
$$

ESAIM: Proc., VoL. 3, 1998, 101-117 
En reportant ces deux équations dans (14) et en tenant compte du fait que la vitesse de la frontière coïncide avec la vitesse du fluide à cet endroit, on obtient

$$
\frac{1}{2} \frac{d}{d t} \int_{\Omega_{F}(t)} \mathbf{u}^{2}+\nu \int_{\Omega_{F}(t)}(\nabla \mathbf{u})^{2}+\int_{\gamma(t)}\left(p \mathbf{n}-\nu \frac{\partial \mathbf{u}}{\partial \mathbf{n}}\right) \mathbf{u}=\int_{\Omega_{F}(t)} \mathbf{f u},
$$

et l'on voit apparaître naturellement des énergies de la partie fluide (énergie cinétique+énergie de dissipation). Pour la partie solide, on agit de même, par exemple pour les équations de l'élasticité linéarisée tridimensionnelle, on multiplie formellement par $\frac{\partial \mathbf{d}}{\partial t}$, il vient après intégration par parties,

$$
\frac{1}{2} \frac{d}{d t} \int_{\hat{\Omega}_{S}} \mathbf{d}^{2}+\frac{1}{2} \frac{d}{d t} a(\mathbf{d}, \mathbf{d})=\int_{\gamma} \sigma(\mathbf{d}) \cdot \mathbf{n} \frac{\partial \mathbf{d}}{\partial t}+\int_{\hat{\Omega}_{S}} \mathrm{~g} \frac{\partial \mathbf{d}}{\partial t},
$$

avec, en utilisant la convention de sommation sur l'indice répété

$$
a(\mathbf{d}, \mathbf{b})=\int_{\hat{\Omega}_{S}} \lambda \varepsilon_{k k}(\mathbf{d}) \varepsilon_{l l}(\mathbf{b})+2 \mu \varepsilon_{i j}(\mathbf{d}) \varepsilon_{i j}(\mathbf{b}) .
$$

On en déduit un bilan d'énergie. En effet, en ajoutant (15) et (16), on obtient, par simplification des termes de contraintes, en utilisant le fait que, sur l'interface $\gamma$ on a $\mathbf{u}(\mathbf{x}+\mathbf{d}(t, \mathbf{x}))=$ $\frac{\partial \mathbf{d}}{\partial t}(t, \mathbf{x})$,

$$
\begin{aligned}
\frac{1}{2} \frac{d}{d t} \int_{\Omega_{F}(t)} \mathbf{u}^{2}+\nu \int_{\Omega_{F}(t)}(\nabla \mathbf{u})^{2} & +\frac{1}{2} \frac{d}{d t} \int_{\hat{\Omega}_{S}}\left(\frac{\partial \mathbf{d}}{\partial t}\right)^{2}+\frac{1}{2} \frac{d}{d t} a(\mathbf{d}, \mathbf{d}) \\
& =\int_{\Omega_{F}(t)} \mathbf{f u}+\int_{\hat{\Omega}_{S}} \mathrm{~g} \frac{\partial \mathbf{d}}{\partial t} .
\end{aligned}
$$

D'où le résultat, en utilisant l'ellipticité de la forme bilinéaire $a$ sur $H_{0, \Gamma_{S}}^{1}\left(\Omega_{S}\right)$ (c.f [10]), où $H_{0, \Gamma_{S}}^{1}\left(\Omega_{S}\right)$ désigne le sous espace de $H_{0}^{1}\left(\Omega_{S}\right)$ des fonctions qui s'annulent sur $\Gamma_{S}$,

Theorème 1 Si la solution du problème couplé existe, son énergie reste bornée au sens suivant:

$$
\begin{aligned}
& \|\mathbf{u}\|_{L^{\infty}\left(0, T ; L^{2}\left(\Omega_{F}(t)\right)\right.}^{2}+\nu\|\mathbf{u}\|_{L^{2}\left(0, T_{;} H^{1}\left(\Omega_{F}(t)\right)\right.} \\
& \left.+\|\mathbf{d}\|_{W^{1, \infty}\left(0, T ; L^{2}\left(\Omega_{S}\right)\right)}+\|\mathbf{d}\|_{L^{\infty}\left(0, T ; H_{0, \Gamma_{S}}\right.}\left(\Omega_{S}\right)\right)
\end{aligned}
$$

On aurait pu faire le même raisonnement avec les équations de plaques, et aurait obtenu également des estimations d'énergie car les termes de couplage, en tenant compte des conditions à l'interface, s'éliminent de la même façon.

Remarque 1 Comme il a déjà été remarqué dans [14], la présence des termes non linéaires (termes de convection) de Navier-Stokes est fondamentale pour satisfaire à la condition de stabilité dans les normes précédentes, dans le cas d'un domaine qui dépend du temps. En effet, sans leur présence, la simplification du terme provenant de la dérivée par rapport au domaine dans la formule de Reynolds ne se fait plus et ce terme cubique, qui n'a pas de signe donné, ne peut être majoré par les autres contributions. Il s'ensuit que le problème risque d'être alors mal posé. Numériquement, on a remarqué des cas d'instabilités lorsque l'on simule le couplage des équations de Stokes (seules) avec les équations de l'élasticité.

À ce jour, l'analyse de tels problèmes de couplage n'est pas définitive. Lorsque le domaine fluide peut être considéré comme fixe au cours du temps (dans le cas où les déformations de la structure sont petites) nous pouvons citer des théorèmes d'existence. En particulier, dans [25], il est démontré l'existence et l'unicité (en dimension 2) de solutions faibles pour 
les équations de Navier-Stokes couplées à celles de l'élasticité linéarisée, avec des conditions de transmissions différentes de celles énoncées précédemment. Des études ont également été faites pour les équations de Navier-Stokes dans des domaines variables en temps, soit dans le cas où cette évolution est connue a priori ([23], [30]), soit pour des problèmes à frontière libre ([1], [5], [31], [32]).

Nous avons démontré [18], [17], que le problème de couplage d'un fluide en dimension 2 d'espace, qui interagit avec un disque rigide, possédant donc 3 degrés de liberté ( 2 translations, $\mathbf{w}_{G}$, et une rotation $\theta$ ) admet au moins une solution. Cette analyse est faite dans des espaces plus réguliers que les espaces d'énergie précédents. Un travail reste donc à faire pour passer de l'existence en temps petit d'une solution régulière à l'existence de solution dans les espaces naturels pour un temps plus long (mais tel que, en particulier, le solide ne touche pas le bord fixe du domaine fluide). L'extension à des situations où le solide dépend de plus de 3 degrés de liberté (mais toutefois en nombre fini) est aussi en cours. Ceci correspond au cas où la déformation du solide est décrit par une combinaison linéaire de ses premiers modes propres.

On a ainsi

Theorème 2 Soit $r$ un réel, $1<r<3 / 2$. On suppose que $\mathbf{u}_{0} \in H^{r+1}(\Omega)$, que $\mathbf{f}$ est suffisamment régulière et que la masse et le moment d'inertie du disque rigide sont assez grands alors il existe $T_{2}>0$ dépendant des données tel que le problème admet une solution avec $\mathbf{u} \in K_{T_{2}}^{r+2}(\Omega), \nabla q \in K_{T_{2}}^{r}(\Omega), \mathbf{w}_{G} \in H^{r / 2+1}\left(0, T_{2}\right)$ et $\theta \in H^{r / 2+2}\left(0, T_{2}\right)$.

L'espace $K_{T_{2}}^{r}(\Omega)$ est défini ici comme

$$
K_{T_{2}}^{r}(\Omega)=L^{2}\left(0, T ; H^{r}(\Omega)\right) \cap H^{r / 2}\left(0, T ; L^{2}(\Omega)\right) .
$$

Pour l'étude de problèmes de couplage fluide structure stationnaires, pour lesquels le domaine fluide est une inconnue, on pourra consulter [17] où il est montré que pour des données (forces extérieures) assez petites, le problème est bien posé.

\section{Formulation ALE pour la simulation numérique}

L'une des difficultés pour la simulation d'interaction fluide structure vient du fait que le domaine fluide bouge. Si l'on prévoit d'utiliser une discrétisation en différences, éléments, volumes finis ou une discrétisation spectrale, il résulte du mouvement du domaine de calcul que les mailles (adaptées à ce domaine) doivent être modifiées au cours du temps ou qu'alors une procédure spéciale doit être développée pour tenir compte d'un maillage (fixe) qui n'est plus adapté au domaine de calcul fluide puisqu'il ne suit pas la déformation de l'interface.

Pour compenser cette défaillance du maillage, une première méthode consiste à utiliser des conditions aux limites dites de transpiration sur cette frontière fixe (qui ne cöncide donc pas avec la frontière réelle du fluide). L'idée est de linéariser les conditions aux limites sur l'interface mobile (supposée proche de la surface fixe) et de prendre ces nouvelles relations comme conditions aux limites sur la surface fixe (voir par exemple [4]). À notre connaissance, cette technique ne se justifie que pour de petites déformations de l'interface. Par ailleurs, toujours pour traiter ce type de problèmes sur des maillages fixes, on a vu récemment apparaitre des techniques de domaines fictifs $([15]),([16])$ qui permettent de traiter les conditions aux limites par l'intermédiaire d'un multiplicateur de Lagrange. On peut finalement penser à une définition particulière des stencils pour les points proches de l'interface, pour l'écriture des schémas aux différences finies comme c'est le cas, par exemple, dans les articles utilisant le concept de lignes de niveau [2]. Ces techniques sont réputées d'ordre relativement bas ou assez couteuses et difficiles à mettre en ouvre. En particulier, une approximation d'ordre suffisamment élevé dans une partie critique du domaine de calcul (couches limites) est assez 
difficile à obtenir. On pourrait aussi penser à utiliser la technique proposée par C. H. Bruneau ([8]), qui consisterait ici à "remplir" la structure d'un fluide fictif régit par des équations de Navier Stokes mais avec une viscosité telle que ce fluide fictif aurait le même mouvement d'ensemble que la structure.

Une seconde approche peut également être envisagée. Il s'agit de se ramener à une écriture des équations sur $\Omega_{F}(0)$. On suppose pour cela qu'une application $\phi(t, \mathbf{x})$ inversible et suffisamment régulière en espace existe entre le domaine fluide initial $\Omega_{F}(0)$ (ou tout autre domaine de référence $\hat{\Omega}_{F}$ ) et $\Omega_{F}(t)$. Cette application permet en fait de rapporter tout le problème sur le domaine de référence $\Omega_{F}(0)$ par changement de variables et l'on peut ainsi résoudre un problème aux dérivées partielles avec des coefficients qui dépendent du temps sur domaine fixe. Il est à noter que si $\phi(t, \mathbf{x})$ désigne la position réelle des particules fluides (c'est-à-dire si $\left.\frac{\partial \phi}{\partial t}(t, \mathbf{x})=\mathbf{u}(t, \phi(t, \mathbf{x}))\right)$ on obtient alors les équations de Navier-Stokes écrites en variables Lagrangiennes. En fait, il est très rare que l'on fasse ce choix, car on arriverait souvent à des équations avec des coefficients très peu réguliers et des systèmes discrets assez complexes à résoudre. Il suffit d'imaginer, en effet, la forme de cette application $\phi$ lorsque l'on est en présence d'un tourbillon au centre du domaine fluide. La seule chose que l'on demande à $\phi$ est de bien représenter la géométrie instationnaire de $\Omega_{F}(t)$. Il suffit, pour cela, que la vitesse du bord soit bien représentée (on pourrait même se contenter d'avoir le déplacement normal bien représenté !) c'est-à-dire que l'on demande à $\phi$ de vérifier

$$
\frac{\partial \phi}{\partial t}(t, \mathbf{x})=\mathbf{u}(t, \phi(t, \mathbf{x})) \text { en tout point } \mathbf{x} \text { de } \partial \Omega_{F}(0)
$$

L'application $\phi$ est donc définie comme un relèvement de la déformation du bord. Ce relèvement peut se faire en résolvant une équation aux dérivées partielles de type Poisson. Cette solution pouvant conduire à des applications $\phi$ non inversibles, on préfère souvent résoudre un problème de Stokes ou de l'élasticité qui, grâce à la condition d'incompressibilité sont plus robustes (c.f. [21], par exemple). On pourra consulter les travaux de Lessoine et Farhat ([24]), par exemple pour d'autres techniques, et en particulier pour une étude sur l'influence de la dynamique de $\phi$ sur la dynamique du système couplé. Le système couplé initial (fluide + structure) en tant que système instationnaire peut, en effet, entrer en résonance et l'échange d'énergie (fluide $\rightarrow$ structure, structure $\rightarrow$ fluide) peut s'amplifier petit à petit et conduire à des problèmes mécaniques sévères (empennages d'avion cassés par exemple). Si, aux équations qui traduisent cette dynamique, on ajoute une autre dynamique (celle du maillage) cet échange d'énergie à deux devient un échange d'énergie à trois et des instabilités fictives ont parfois été notées.

En fait, rares sont les approches suivant la description précédente. En effet, cette première description conduit à la résolution d'équations avec des coefficients non constants, donc à une difficulté à évaluer les différentes matrices de masse et de rigidité. Elle conduit aussi à des schémas avec des stencils assez larges. Surtout pour les applications en dimension 3, cela peut vite devenir un problème. Une autre raison pour ne pas travailler sur le domaine de référence vient du fait qu'en mécanique, en particulier pour les fluides, certaines quantités doivent être conservées et cette conservation est plus simple à imposer et à vérifier sur les équations eulériennes. Il faut alors revenir à une discrétisation des équations sur le domaine $\Omega_{F}(t)$, et donc obtenir un maillage de $\Omega_{F}(t)$, pour tout temps. La tendance actuelle est d'utiliser des approches arbitraires lagrangiennes eulériennes (A.L.E.) qui permettent en quelque sorte de travailler automatiquement sur un maillage qui se déforme avec le domaine fluide. Ces techniques reposent sur la notion de vitesse de maillage et de caractéristiques. Il suffit bien entendu d'utiliser le maillage de $\Omega_{F}(0)$ et l'application $\phi(t, \mathbf{x})$, précédemment introduite. On comprend pourquoi il est préférable de ne pas suivre les particules fluides pour définir $\phi$ mais seulement le bord du domaine si l'on souhaite avoir à ce stade un maillage correct du domaine $\Omega_{F}(t)$ ! 
Pour définir les dérivations par rapport à la variable d'espace, il suffit alors de reconstruire les matrices élémentaires sur un maillage (structuré ou non) déformé, et ce, à chaque pas de temps. Pour contrer tout argument de surcoût qui pourrait être opposé à cette dernière approche, il suffit de noter que, en dimension 3, avec des géométries stationnaires mais complexes, il arrive très souvent que les matrices de masse et de rigidité soient reconstruites à chaque pas de temps afin de diminuer le stockage en mémoire vive (la géométrie tient en mémoire vive mais pas les matrices). Le surcoût de construction des matrices est donc relativement faible.

Si la difficulté de la définition des dérivations spatiales est ainsi aplanie, la dérivation temporelle reste une difficulté puisque, basée sur une approche différence finie, elle consiste à faire la différence des vitesses en deux temps proches et en un même point. Le problème vient des points proches de l'interface puisque rien n'assure que du fluide se trouve bien en présence à ces deux instants en ce point là! De plus on préfêre souvent utiliser des points de maillages pour éviter les interpolations d'un maillage (celui au temps $t^{n+1}$ ) sur un autre (celui au temps $t^{n}$ ). La technique ALE consiste alors à travailler sur le seul maillage $\Omega_{F}\left(t^{n+1}\right)$ (ou $\Omega_{F}\left(t^{n}\right)$ ) en utilisant par exemple l'application $\phi\left(t^{n+1},.\right) \circ\left(\phi\left(t^{n}, .\right)\right)^{-1}$.

En définissant les variables caractéristiques $X(x, s ; t)$ par

$$
X(x, s ; t)=\phi(s, .) \circ(\phi(t, x))^{-1},
$$

et, pour $t$ fixé, la vitesse $\mathbf{v}$ en ces points

$$
\mathbf{v}(s, x)=\mathbf{u}(s, X(x, s ; t)),
$$

on remarque que, en tout point $x$ de $\Omega_{F}(t)$ et tout temps $s$

$$
\frac{\partial \mathbf{v}}{\partial s}(s, x)=\frac{\partial \mathbf{u}}{\partial s}(s, X(x, s ; t))+\mathbf{c} \nabla \mathbf{u}(s, X(x, s ; t)),
$$

où $\mathbf{c}$ désigne la vitesse de grille

$$
\mathbf{c}(s, X(x, s ; t))=\frac{\partial X}{\partial s}(x, s ; t) .
$$

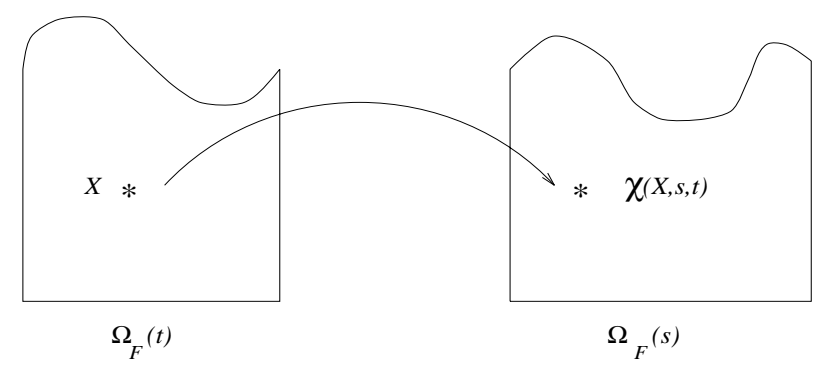

En reportant cette égalité dans les équations de Navier-Stokes (1) écrites au temps $s$ et au point $X(x, s ; t)$, il vient pour $x \in \Omega_{F}(t)$

$$
\begin{aligned}
& \frac{\partial \mathbf{v}}{\partial s}(s, x)-\nu \Delta \mathbf{u}(s, X(x, s ; t)) \\
& +(\mathbf{u}-\mathbf{c}) \nabla \mathbf{u}(s, X(x, s ; t))+\nabla p(s, X(x, s ; t))=\mathbf{f}(s, X(x, s ; t)) .
\end{aligned}
$$

C'est cette dernière formulation qui est appelée formulation ALE des équations fluides. On a ainsi résolu le problème de dérivation en temps, puisque l'on considère maintenant la fonction $\mathbf{v}$ qui est définie sur le domaine fixe $\Omega_{F}(t)$ (on rappelle que $t$ est momentanément fixé et $s$ 
est variable). Par exemple, pour un schéma en temps d'ordre 1 on propose, pour approcher la solution de définir une suite de vitesses $\mathbf{u}^{n} \equiv \mathbf{u}\left(t^{n},.\right)$ et de pression $p^{n} \equiv p\left(t^{n},.\right)$ définies dans $\Omega_{F}\left(t^{n+1}\right)$ selon un schéma qui sera du type

$$
\begin{aligned}
& \frac{\mathbf{u}^{n+1}(x)-\mathbf{u}^{n}\left(X\left(x, t^{n} ; t^{n+1}\right)\right)}{t^{n+1}-t^{n}}-\nu \Delta \mathbf{u}^{n+1}\left(X\left(x, t^{n+1} ; t^{n+1}\right)\right) \\
& +\left(\mathbf{u}^{n}-\mathbf{c}^{n}\right) \nabla \mathbf{u}^{n}\left(X\left(x, t^{n} ; t^{n+1}\right)\right)+\nabla p^{n+1}\left(X\left(x, t^{n+1} ; t^{n+1}\right)\right)=\mathbf{f}^{n+1}\left(X\left(x, t^{n+1} ; t^{n+1}\right)\right) .
\end{aligned}
$$

On rappelle alors que $X\left(x, t^{n+1} ; t^{n+1}\right)=x$ et on remarque que $X\left(x, t^{n} ; t^{n+1}\right)=x+\mathcal{O}\left(t^{n+1}-\right.$ $\left.t^{n}\right)$ qui nous conduit donc au schéma

$$
\begin{aligned}
& \frac{\mathbf{u}^{n+1}(x)-\mathbf{u}^{n}\left(X\left(x, t^{n} ; t^{n+1}\right)\right)}{t^{n+1}-t^{n}}-\nu \Delta \mathbf{u}^{n+1}(x) \\
& +\left(\mathbf{u}^{n}-\mathbf{c}^{n}\right) \nabla \mathbf{u}^{n}(x)+\nabla p^{n+1}(x) \quad=\mathbf{f}^{n+1}(x) \quad \text { dans } \Omega_{F}\left(t^{n+1}\right),
\end{aligned}
$$

auquel on doit ajouter la condition d'incompressibilité sur $\mathbf{u}^{n+1}$.

On remarque donc que pour la définition d'un schéma à l'ordre 1 la procédure précédente est l'une des solutions possibles. On doit, en effet, être capable de calculer $\left(\nabla \mathbf{u}^{n}\right)\left(X\left(x, t^{n}\right.\right.$; $\left.t^{n+1}\right)$ ) et l'intégrer avec des fonctions tests définies sur $\Omega_{F}\left(t^{n}\right)$. Il y a donc des Jacobiens de transformation $x \mapsto X$ à faire intervenir. Si à l'ordre 1 ces Jacobiens sont égaux à 1, il n'en est plus de même à l'ordre 2 .

\section{Analyse de procédures de découplage}

Comme nous l'avons déjà annoncé, le cadre dans lequel nous envisageons la simulation des phénomènes d'interaction fluide structure est le suivant: on suppose que l'on dispose de deux codes, un permettant de simuler le comportement du fluide sur des domaines qui se déforment au cours du temps (qui utilisent, par exemple, la formulation ALE décrite précédemment), un autre permettant de simuler le comportement de la structure. Pour ce dernier il s'agit, en général, d'un code lagrangien. Ce qui nous intéresse ici est l'obtention d'algorithmes de couplage efficaces de ces deux codes. On peut envisager plusieurs stratégies afin de discrétiser en temps les conditions de couplages, stratégies qui vont du totalement implicite au totalement explicite. Dans le cas où la structure et le fluide sont modélisés par des équations posées sur des domaines ayant la même dimension (3D-3D, par exemple), on peut dénombrer, au moins, 11 stratégies différentes.

Supposons que l'on connaisse les approximations de la vitesse, de la pression, du déplacement et de la géométrie à l'instant $n \Delta t$, où $\Delta t$ désigne le pas de discrétisation en temps. La première stratégie que l'on peut envisager est celle où la géométrie et les conditions de couplage sont traitées implicitement à chaque itération en temps. En particulier, l'égalité des vitesses et l'égalité des contraintes ont lieu à l'instant $(n+1) \Delta t$ sur un domaine $\Omega^{n+1}$ déterminé par le déplacement à l'instant $(n+1) \Delta t$. Cela conduit à un algorithme qui conserve toutes les non linéarités du problème initial et qui peut être résolu itérativement. Dans [28], les auteurs ont proposé une méthode itérative qui consiste à résoudre les équations fluides avec des conditions de Dirichlet, les équations de structure avec des conditions de Neumann calculées à partir de la résolution précédente et mettre à jour la position de l'interface en utilisant un paramètre de relaxation convenablement choisi. Une autre stratégie consiste à traiter le comportement de la frontière explicitement et les conditions de couplage implicitement. On commence donc, connaissant les approximations de la vitesse, de la pression, du déplacement et de la géométrie aux instants précédents, par extrapoler la géométrie. Puis, on résout les équations du fluide et de la structure de façon non décalée, de sorte que les vitesses et les contraintes à l'interface sont égales à l'instant $(n+1) \Delta t$. Là encore l'égalité des vitesses discrètes et des contraintes discrètes à l'instant $(n+1) \Delta t$ peut être obtenu en itérant. Plusieurs choix sont possibles pour résoudre les équations fluides: on peut, par exemple, 
choisir de les résoudre sur la configuration à l'instant $n \Delta t$, ou bien sur la nouvelle géométrie prédite lors de l'étape d'extrapolation. Une troisième classe de schémas est obtenue en explicitant toutes les conditions de couplage. Ceci conduit à des schémas décalés, c'est-à-dire des schémas où les résolutions des équations du fluide et de la structure se font l'une après l'autre et une seule fois par pas de temps. Le traitement des conditions de couplages est alors explicite. On peut envisager de résoudre, d'abord la partie fluide avec des conditions de Dirichlet (vitesse donnée) extrapolée à partir des étapes précédentes, ce qui nous permet de calculer les contraintes $\sigma_{F}^{n+1}$ appliquées par le fluide sur la structure à l'instant $(n+1) \Delta t$. Les équations de structure sont alors traitées avec des conditions de Neumann (contraintes données à l'interface). Une autre option est de calculer la vitesse et la pression du fluide avec des conditions de Neumann (CN) à l'interface, provenant du tenseur des contraintes du matériau $\sigma_{S}$ calculé aux étapes précédentes, puis d'en déduire le déplacement de la structure à l'instant $(n+1) \Delta t$ en mettant comme conditions aux limites des conditions de Dirichlet (CD), venant de la partie fluide que l'on vient juste de résoudre. Nous pouvons également commencer par résoudre d'abord les équations de structure puis les équations fluides (il ne s'agit pas seulement d'un décalage d'indices) avec les mêmes possibilités pour les conditions aux limites. Dans tous les cas présentés précédemment, nous avons la possibilité de résoudre les équations qui régissent l'écoulement fluide soit sur le domaine à l'instant $n \Delta t$, soit sur le domaine à l'instant $(n+1) \Delta t$. En résumé, si l'on note $U^{n}, P^{n}, D^{n}, \Omega^{n}$ les approximations de la vitesse, de la pression, du déplacement et de la géométrie à l'instant $n \Delta t$, si $F$ désigne la partie fluide et $S$ la partie structure, nous avons

1. Traitement implicite de la géométrie.

Traitement implicite des conditions de couplage.

$\Longrightarrow U^{n+1}, P^{n+1}, D^{n+1}, \Omega^{n+1}$

2. Extrapolation de la géométrie $\Longrightarrow \Omega^{n+1}$.

Traitement implicite des conditions de couplage $\Longrightarrow U^{n+1}, P^{n+1}, D^{n+1}$.

3. Extrapolation de la géométrie $\Longrightarrow \Omega^{n+1}$.

Traitement explicite des conditions de couplage:

(a)

$$
F^{n+1}+C D^{n} \longrightarrow\left(U^{n+1}, P^{n+1}\right) \longrightarrow \sigma_{F}^{n+1} \longrightarrow S^{n+1}+C N^{n+1} \longrightarrow D^{n+1},
$$

$$
F^{n+1}+C N^{n} \longrightarrow\left(U^{n+1}, P^{n+1}\right) \longrightarrow S^{n+1}+C D^{n+1} \longrightarrow D^{n+1} \longrightarrow \sigma_{S}^{n+1} .
$$

On peut encore envisager de commencer par la structure soit

(c)

$$
S^{n+1}+C D^{n} \longrightarrow D^{n+1} \longrightarrow \sigma_{S}^{n+1} \longrightarrow F^{n+1}+C N^{n+1} \longrightarrow\left(U^{n+1}, P^{n+1}\right),
$$

$$
S^{n+1}+C N^{n} \longrightarrow D^{n+1} \longrightarrow F^{n+1}+C D^{n+1} \longrightarrow\left(U^{n+1}, P^{n+1}\right) \longrightarrow \sigma_{F}^{n+1} .
$$

Pour les stratégies 2 et 3 , on peut, à chaque fois, résoudre les équations fluides sur $\Omega^{n}$ ou $\operatorname{sur} \Omega^{n+1}$.

Certaines procédures de découplage ont déjà été étudiées. On peut citer les travaux de Piperno, Farhat \& Larrouturou ([29]) dans lesquels il est présenté une analyse linéaire du couplage d'un piston mobile et d'un fluide compressible. La stratégie 3.(d) est analysée et une méthode de prédiction-correction doit être associée pour assurer la stabilité du schéma. À ces 11 procédures s'ajoutent donc encore des procédures à plusieurs sous pas de temps du 
type prédiction correction. Dans [20], nous avons étudié, sur un modèle monodimensionnel, trois de ces stratégies, qui correspondent aux cas 2, 3.(a) et 3.(b). Dans chacun des cas, les équations de la partie fluide sont écrites en formulation ALE, et la vitesse à l'instant $(n+1) \Delta t$ est calculée sur la configuration à l'instant $n \Delta t$. La géométrie est extrapolée à l'ordre 1 et nous avons considéré des schémas d'ordre 1 en temps pour les équations fluides et pour les équations de structure. Nous montrons que le schéma correspondant au cas 2 est stable, consistant, et convergent avec un taux de convergence $\Delta t^{3 / 4}$. Pour les deux autres, seule une analyse de stabilité est effectuée qui fait intervenir, en outre, dans le cas 3.(b) une discrétisation éléments finis en espace (on a, en effet, besoin d'une condition de type CFL liant le pas de discrétisation en temps et les pas de maillage en espace). Numériquement, il semble néanmoins que ces deux derniers schémas se comportent de la même façon.

\section{Analyse de la discrétisation en espace}

Outre la difficulté liée à la discrétisation en temps, se pose, quand on a deux codes basés sur des discrétisations spatiales différentes, le problème d'assurer entre les fonctions discrètes de la partie fluide et les fonctions discrètes de la partie structure les continuités nécessaires.

$\mathrm{Si}$ l'on traite, et c'est le cadre dans lequel nous nous placerons, les discrétisations sous forme variationnelle, les conditions de raccord sur les contraintes s'effectuent, en fait, par l'intermédiaire du transposé de l'opérateur de raccord des fonctions tests. Il semble naturel, pour avoir les estimations d'énergie a priori valables dans le cas continu, d'imposer la même condition de raccord sur les fonctions tests que sur les fonctions d'essai. Supposons que l'on ait des discrétisations en espace de type éléments finis. Soit $h$ le pas de maillage associé à la structure, et $H$ celui associé au fluide. On note $Y_{h}$ l'espace discret associé à la partie structure. Pour simplifier, nous allons supposer que les déplacements sont petits et donc que $\Omega_{F}(t)=\Omega_{F}$, et se restreindre aux équations de Stokes (ce qui assure l'existence de solutions faibles). On note, d'autre part, $X_{H} \times M_{H}$ les espaces discrets associés à la vitesse et à la pression du fluide.

- Formulation variationnelle du problème continu.

$$
\begin{aligned}
& \int_{\Omega_{F}} \frac{\partial \mathbf{u}}{\partial t} \mathbf{v}+\nu \int_{\Omega_{F}} \nabla \mathbf{u} \nabla \mathbf{v}+\int_{\Omega_{F}} p \nabla \cdot \mathbf{v}+\int_{\hat{\Omega}_{S}} \frac{\partial^{2} \mathbf{d}}{\partial t^{2}} \mathbf{b}+a(\mathbf{d}, \mathbf{b}) \\
& =\int_{\Omega_{F}} \mathbf{f v}, \forall(\mathbf{v}, \mathbf{b}) \in \mathcal{V}, \\
& \int_{\Omega_{F}} \nabla \cdot \mathbf{u} \mu=0, \forall \mu \in M,
\end{aligned}
$$

où $\mathcal{V}$ est défini par

$$
\mathcal{V}=\{(\mathbf{v}, \mathbf{b}) \in X \times Y / \mathbf{v}(\mathbf{x})=\mathbf{b}(\mathbf{x}), \forall \mathbf{x} \in \gamma\},
$$

et $\gamma$ est l'interface (supposée fixe) de couplage.

- Formulation variationnelle du problème discret.

Trouver $\left(\mathbf{u}_{H}, \mathbf{d}_{h}\right)$ tel que $\mathbf{u}_{H}(\mathbf{x})=\Pi_{H}\left(\frac{\partial \mathbf{d}_{h}}{\partial t}\right)(\mathbf{x}), \forall \mathbf{x} \in \gamma$

$$
\begin{aligned}
& \int_{\Omega_{F}} \frac{\partial \mathbf{u}_{H}}{\partial t} \mathbf{v}_{H}+\nu \int_{\Omega_{F}} \nabla \mathbf{u}_{H} \nabla \mathbf{v}_{H}+\int_{\Omega_{F}} p_{H} \nabla \cdot \mathbf{v}_{H} \\
& +\int_{\Omega_{S}} \frac{\partial^{2} \mathbf{d}_{h}}{\partial^{2} t} \mathbf{b}_{h}+a\left(\mathbf{d}_{h}, \mathbf{b}_{h}\right)=\int_{\Omega_{F}} \mathbf{f v}_{H} \\
& \forall\left(\mathbf{v}_{H}, \mathbf{b}_{h}\right) \in \mathcal{V}_{H, h}, \\
& \int_{\Omega_{F}} \nabla \cdot \mathbf{u}_{H} \mu_{H}=0, \forall \mu_{H} \in M_{H}
\end{aligned}
$$


où $\mathcal{V}_{H, h}$ est défini par

$$
\mathcal{V}_{H, h}=\left\{\left(\mathbf{v}_{H}, \mathbf{b}_{h}\right) \in X_{H} \times Y_{h} / \mathbf{v}_{H}(\mathbf{x})=\Pi_{H}\left(\mathbf{b}_{h}(\mathbf{x})\right), \forall \mathbf{x} \in \gamma\right\} .
$$

L'opérateur $\Pi_{H}$ désigne un opérateur de raccord. Il est alors clair que $\left(\mathbf{u}_{H}, \Pi_{H}\left(\frac{\partial \mathbf{d}_{h}}{\partial t}\right)\right)$ sont des fonctions tests admissibles. On obtient, ainsi, du moins formellement, des estimations d'énergie discrètes, comme dans le cas continu. On cherche à décrire les propriétés de l'opérateur $\Pi_{H}$ afin d'obtenir des estimations d'erreur optimales. Puisque l'opérateur $\Pi_{H}$ est indépendant du temps nous pouvons, dans un premier temps, focaliser notre attention sur l'étude de problèmes stationnaires. Nous cherchons un opérateur conduisant à des estimations d'erreur sur le système couplé de la forme

$$
\left\|\mathbf{u}-\mathbf{u}_{H}\right\|_{X}+\left\|\mathbf{d}-\mathbf{d}_{h}\right\|_{Y} \leq C\left(H^{\alpha}+h^{\beta}\right) .
$$

Elles sont dites optimales si l'exposant $\alpha$ (resp. $\beta$ ) correspond à des estimations d'erreur optimales lorsque l'on regarde la partie fluide (resp. structure) seule. En fait, en considérant un problème stationnaire, nous avons rapproché l'étude de ce problème de celui du couplage de discrétisations non conformes dans le cas des décompositions de domaines sans recouvrement. Dans cette voie, la méthode des éléments avec joints a été proposée dans [7] pour conduire justement à des estimations d'erreur optimales. Dans la méthode des éléments avec joints, le raccord faible des fonctions discrètes se fait par un raccord intégral. L'opérateur $\Pi_{H}$ à valeur dans l'espace des traces $W_{H}$ de $X_{H}$ sur $\gamma$ est alors défini par:

$$
\int_{\gamma}\left(\mathbf{b}-\Pi_{H}(\mathbf{b})\right) \psi=0, \quad \forall \psi \in \tilde{W}_{H}
$$

où $\tilde{W}_{H}$ est, en dimension 2 d'espace, un sous espace de codimension 2 de $W_{H}$.

Nous avons montré dans [19], que le raccordement ponctuel par interpolation (i.e. $\mathbf{u}_{H}(\mathbf{x})=$ $\frac{\partial \mathbf{d}_{h}}{\partial t}(\mathbf{x})$ en tout point $\mathbf{x}$ correspondant aux degrés de liberté fluide), dans le cas du couplage du type fluide 2D - structure 1D, permet d'avoir le plus souvent l'optimalité de l'approximation. Cependant, en particulier pour une approximation d'ordre 2 (éléments finis $P_{2}$ ) en espace pour la vitesse lorsque l'opérateur régissant le comportement de la structure est d'ordre 2, alors la méthode de raccordement ponctuel précédente n'est plus optimale, contrairement à la méthode des joints, qui elle donne toujours des approximations optimales. Nous pouvons, pour ces deux types de raccords, résumer les résultats obtenus dans le tableau suivant:

\begin{tabular}{|c|c|c|c|}
\hline & $\begin{array}{c}\text { "Structure 1D" } \\
\text { opérateur ordre 2 }\end{array}$ & $\begin{array}{c}\text { "Structure 1D" } \\
\text { opérateur ordre 4 }\end{array}$ & $\begin{array}{c}\text { "Structure 2D" } \\
\text { (élasticité) }\end{array}$ \\
\hline $\begin{array}{c}\text { "Fluide" 2D } P_{k} \\
+\end{array}$ & $\begin{array}{c}k=1 \text { optimal } \\
\text { interpolation }\end{array}$ & $\begin{array}{c}k \leq 2 \text { optimal } \\
k>1 \text { non optimal }\end{array}$ & non optimal \\
\hline $\begin{array}{c}k>2 \text { non optimal } \\
\text { "Fluide" 2D } P_{k} \\
+\end{array}$ & optimal $\forall k$ & optimal $\forall k$ & optimal $\forall k$ \\
méthode des joints & & & \\
\hline
\end{tabular}

Les mêmes conclusions sont valables en $3 D$. Afin de comprendre les raisons essentielles de ce comportement, nous proposons dans la section qui suit une étude simplifiée sur un problème stationnaire.

\section{6 Étude simplifiée du couplage non conforme}

On considère $\Omega$ un ouvert borné, et $\Gamma$ une partie non vide connexe du bord. On pose $\Gamma_{0}=\partial \Omega \backslash \Gamma$. Nous considérons le problème suivant: 
trouver $u$ et $d$ tels que

$$
\begin{aligned}
& \left\{\begin{array}{lll}
\Delta u & =f & \text { dans } \Omega, \\
u & =0 & \text { sur } \partial \Omega,
\end{array}\right. \\
& \left\{\begin{array}{l}
\Delta d=g-\frac{\partial u}{\partial n} \quad \text { sur } \Gamma, \\
\left.d\right|_{\partial \Gamma}=0 .
\end{array}\right.
\end{aligned}
$$

Les deux équations (23) et (24) sont maintenant découplées et linéaires. Connaissant $u$ nous pouvons calculer sa dérivée normale et en déduire $d$. Nous allons réécrire ces équations sous forme variationnelle. On pose

$$
\mathcal{V}=\left\{(w, b) \in H_{0, \Gamma_{0}}^{1}(\Omega) \times H_{0}^{1}(\Gamma) /\left.w\right|_{\Gamma}=b\right\} .
$$

où $H_{0, \Gamma_{0}}^{1}(\Omega)$ est le sous espace de éléments de $H^{1}(\Omega)$ qui s'annulent sur $\Gamma_{0}$. Le problème s'écrit alors

trouver $(u, d) \in H_{0}^{1}(\Omega) \times H_{0}^{1}(\Gamma)$ tels que

$$
\int_{\Omega} \nabla u \nabla w+\int_{\Gamma} \nabla d \nabla b=\int_{\Omega} f w+\int_{\Gamma} g b, \quad \forall(w, b) \in \mathcal{V}
$$

Ce problème est un problème bien posé comme on peut facilement le montrer en vérifiant des conditions inf-sup données dans les hypothèses du théorème 5.2 .1 p: 112 de [3]. On désigne par $\delta$ un paramètre de discrétisation. On considère $X_{\delta}$ un sous espace de dimension finie de $H_{0, \Gamma_{0}}^{1}(\Omega) \cap C^{0}(\bar{\Omega})$ et $Y_{\delta}$ un sous espace de dimension finie de $H_{0}^{1}(\Gamma) \cap C^{0}(\bar{\Gamma})$. L'espace $X_{\delta}^{0}$ désigne l'intersection de $X_{\delta}$ et de $H_{0}^{1}(\Omega)$. D'autre part, on note $W_{\delta}(\Gamma)$ l'espace des traces des éléments de $X_{\delta}$ sur $\Gamma$. On suppose que $W_{\delta}(\Gamma)$ ne contient pas $Y_{\delta}$, ce qui interdit donc l'égalité exacte entre les fonctions test $\left(\left.w\right|_{\Gamma}=b\right)$. L'espace discret associé à $\mathcal{V}$ est noté $\mathcal{V}_{\delta}$, il n'est pas inclus dans $\mathcal{V}$ puisque l'on considère ici une discrétisation non conforme. L'espace $\mathcal{V}_{\delta}$ est défini par:

$$
\mathcal{V}_{\delta}=\left\{\left(w_{\delta}, b_{\delta}\right) \in X_{\delta} \times Y_{\delta} /\left.w_{\delta}\right|_{\Gamma}=\Pi_{\delta}\left(b_{\delta}\right)\right\},
$$

où l'opérateur $\Pi_{\delta}$ désigne un opérateur de raccord faible des fonctions tests discrètes (interpolation ou projection de la méthode des joints). Le problème discret s'écrit alors:

Trouver $\left(u_{\delta}, d_{\delta}\right) \in X_{\delta} \times Y_{\delta}$ tel que

$$
\int_{\Omega} \nabla u_{\delta} \nabla w_{\delta}+\int_{\Gamma} \nabla d_{\delta} \nabla b_{\delta}=\int_{\Omega} f w_{\delta}+\int_{\Gamma} g b_{\delta}, \quad \forall\left(w_{\delta}, b_{\delta}\right) \in \mathcal{V}_{\delta} .
$$

Si l'opérateur $\Pi_{\delta}$ est stable en norme $H^{1}$ nous pouvons démontrer qu'il existe une solution de (26) en reprenant la même démarche que pour le problème continu.

Nous allons étudier l'erreur entre la solution de (25) et la solution discrète de (26), et obtenir un résultat semblable au lemme de Strang ([9]). Nous allons montrer que l'erreur commise peut être majorée par la somme de l'erreur de la meilleure approximation et de l'erreur de consistance. On pose

$$
\begin{aligned}
a(u, w) & =\int_{\Omega} \nabla u \nabla w . \\
\beta(d, b) & =\int_{\Gamma} \nabla d \nabla b .
\end{aligned}
$$

Alors,

$$
a(u, w)=\int_{\Omega} f w, \quad \forall w \in H_{0}^{1}(\Omega)
$$

et

$$
a\left(u_{\delta}, w_{\delta}\right)=\int_{\Omega} f w_{\delta}, \quad \forall w_{\delta} \in X_{\delta}^{0}
$$


Donc nous obtenons, d'après le Lemme de Céa classique

$$
\left\|u-u_{\delta}\right\|_{H_{0}^{1}(\Omega)} \leq C \inf _{v_{\delta} \in X_{\delta}^{0}}\left\|u-v_{\delta}\right\|_{H_{0}^{1}(\Omega)}
$$

Pour la partie monodimensionnelle, nous avons

$$
\beta(d, b)=\int_{\Gamma}\left(g-\frac{\partial u}{\partial n}\right) b, \quad \forall b \in H_{0}^{1}(\Gamma),
$$

et

$$
\beta\left(d_{\delta}, b_{\delta}\right)=\int_{\Gamma} g b_{\delta}+\int_{\Omega} f w_{\delta}-a\left(u_{\delta}, w_{\delta}\right), \quad \forall\left(w_{\delta}, b_{\delta}\right) \in \mathcal{V}_{\delta}
$$

$\mathrm{Or}$

$$
\int_{\Omega} f w_{\delta}=a\left(u, w_{\delta}\right)-\int_{\Gamma} \frac{\partial u}{\partial n} w_{\delta}, \quad \forall w_{\delta} \in X_{\delta}
$$

donc

$$
\beta\left(d_{\delta}, b_{\delta}\right)=\int_{\Gamma} g b_{\delta}+a\left(u-u_{\delta}, w_{\delta}\right)-\int_{\Gamma} \frac{\partial u}{\partial n} w_{\delta}
$$

En retranchant $(27)$ à $(28)$, il vient

$$
\beta\left(d_{\delta}-d, b_{\delta}\right)+a\left(u_{\delta}-u, w_{\delta}\right)=\int_{\Gamma} \frac{\partial u}{\partial n}\left(b_{\delta}-w_{\delta}\right) .
$$

Ainsi pour tout $\left(v_{\delta}, k_{\delta}\right) \in X_{\delta}^{0} \times Y_{\delta}$ nous avons

$\beta\left(d_{\delta}-k_{\delta}, b_{\delta}\right)+a\left(u_{\delta}-v_{\delta}, w_{\delta}\right)=\beta\left(d-k_{\delta}, b_{\delta}\right)+a\left(u-v_{\delta}, w_{\delta}\right)+\int_{\Gamma} \frac{\partial u}{\partial n}\left(b_{\delta}-w_{\delta}\right), \quad \forall\left(w_{\delta}, b_{\delta}\right) \in \mathcal{V}_{\delta}$

Par conséquent, les conditions inf-sup sur $a$ et $\beta$ permettent d'obtenir

$$
\begin{aligned}
\left\|d_{\delta}-d\right\|\left\|_{H_{0}^{1}(\Gamma)}+\right\| u_{\delta}-u \|_{H_{0}^{1}(\Omega)} \leq & C\left[\inf _{b_{\delta} \in Y_{\delta}}\left\|d-b_{\delta}\right\|_{H_{0}^{1}(\Gamma)}+\inf _{w_{\delta} \in X_{\delta}^{0}}\left\|u-w_{\delta}\right\|_{H_{0}^{1}(\Omega)}\right. \\
& \left.+\sup _{\left(w_{\delta}, b_{\delta}\right) \in \mathcal{V}_{\delta}} \frac{\int_{\Gamma} \frac{\partial u}{\partial n}\left(b_{\delta}-w_{\delta}\right)}{\left\|\left(w_{\delta}, b_{\delta}\right)\right\|_{H^{1}(\Omega) \times H^{1}(\Gamma)}}\right]
\end{aligned}
$$

Les deux premiers termes mesurent l'erreur de la meilleure approximation et troisième mesure l'erreur due au raccordement de maillages incompatibles, appelée erreur de consistance.

$$
\sup _{\left(w_{\delta}, b_{\delta}\right) \in \mathcal{V}_{\delta}} \frac{\int_{\Gamma} \frac{\partial u}{\partial n}\left(b_{\delta}-w_{\delta}\right)}{\left\|\left(w_{\delta}, b_{\delta}\right)\right\|_{H^{1}(\Omega) \times H^{1}(\Gamma)}}=\sup _{\left(w_{\delta}, b_{\delta}\right) \in \mathcal{V}_{\delta}} \frac{\int_{\Gamma} \frac{\partial u}{\partial n}\left(b_{\delta}-\Pi_{\delta}\left(b_{\delta}\right)\right)}{\left\|\left(w_{\delta}, b_{\delta}\right)\right\|_{H^{1}(\Omega) \times H^{1}(\Gamma)}} .
$$

Choisissons une discrétisation éléments finis pour la partie bidimensionnelle, en particulier considérons des éléments finis $P_{k}$ Lagrange. Alors un raccord ponctuel (interpolation) donne, pour l'erreur de consistance la majoration suivante

$$
\sup _{\left(w_{\delta}, b_{\delta}\right) \in \mathcal{V}_{\delta}} \frac{\int_{\Gamma} \frac{\partial u}{\partial n}\left(b_{\delta}-w_{\delta}\right)}{\left\|\left(w_{\delta}, b_{\delta}\right)\right\|_{H^{1}(\Omega) \times H^{1}(\Gamma)}} \leq C h \sup _{\left(w_{\delta}, b_{\delta}\right) \in \mathcal{V}_{\delta}} \frac{\left\|b_{\delta}\right\|_{H_{0}^{1}(\Gamma)}}{\left\|\left(w_{\delta}, b_{\delta}\right)\right\|_{H^{1}(\Omega) \times H^{1}(\Gamma)}} \leq C h .
$$

Ainsi, pour $k=1$ la méthode est optimale. Par contre si $k>1$, le raccord ponctuel ne donne plus d'estimation optimale. La méthode des joints permet alors de retrouver le même taux 
de convergence que dans le cas conforme. En effet, revenant à (29) et utilisant la définition de l'opérateur de projection $\Pi_{\delta}$ de $(22)$, il vient

$$
\sup _{\left(w_{\delta}, b_{\delta}\right) \in \mathcal{V}_{\delta}} \frac{\int_{\Gamma} \frac{\partial u}{\partial n}\left(b_{\delta}-\Pi_{\delta}\left(b_{\delta}\right)\right)}{\left\|\left(w_{\delta}, b_{\delta}\right)\right\|_{H^{1}(\Omega) \times H^{1}(\Gamma)}}=\sup _{\left(w_{\delta}, b_{\delta}\right) \in \mathcal{V}_{\delta}} \frac{\int_{\Gamma}\left[\frac{\partial u}{\partial n}-\psi\right]\left(b_{\delta}-\Pi_{\delta}\left(b_{\delta}\right)\right)}{\left\|\left(w_{\delta}, b_{\delta}\right)\right\|_{H^{1}(\Omega) \times H^{1}(\Gamma)}},
$$

pour toute fonction $\psi$ de $\tilde{W}_{H}$. Il suffit alors de choisir $\psi$ comme la meilleure approximation de $\frac{\partial u}{\partial n}$ en norme $L^{2}$ pour obtenir l'optimalité de l'approximation.

On rappelle que si l'on considère un problème elliptique modèle du second ordre que l'on cherche à discrétiser en utilisant une méthode de décomposition de domaine sans recouvrement, le raccord ponctuel donne une estimation d'erreur non optimale. Pour un tel problème, les fonctions tests sont en effet seulement $H^{1 / 2}$ du bord comme trace de fonctions

$H^{1}$, contrairement aux cas étudiés précédemment où elles sont plus régulières puisque solution d'un problème d'ordre 2 "sur le bord". C'est cette régularité de l'opérateur d'ordre 2 pour la partie 1D, qui permet d'avoir une majoration de l'erreur de consistance optimale, pour un raccord ponctuel, dans le cas où le degré $k$ des polynômes associés au fluide égal 1.

\section{Références}

[1] G. AlLain Un problème de Navier-Stokes avec surface libre Thèse de troisième cycle de l'Université Paris VI, 1983

[2] C.ANDERSON et A. Li Integral Equation Preconditioning for the Solution of Poisson's Equation on Geometrically Complex Region Recent UCLA Computational and Applied Mathematics Reports 97.25

[3] I. BABUŠKa Survey Lectures on the Mathematical Foundations of the Finite Element Method. The Mathematical Foundations of the Finite Element Method with application to the partial differential Equations, Ed. By A. K. Aziz, 1972

[4] C.Bardos et O. Pironneau Petites perturbations et équations d'Euler pour l'aéroélasticité. $\mathrm{M}^{2} \mathrm{AN}, \mathrm{vol} 28,4,1994$

[5] J.T. BEALE The Initial Value Problem for the Navier-Stokes Equation with a Free Surface. Comm. on Pure and Applied Mathematics, vol XXXIV, pp 359-392, 1981

[6] M. Bernadou Méthodes d'éléments finis pour les problèmes de coques minces. RMA, Masson, 1994

[7] C. Bernardi, Y. Maday, A. T. Patera A New Non Conforming Approach to Domain Decomposition: the Mortar Element Method. Collège de France Seminar, Pitman, H Brezis, J.L. Lions, 1990

[8] C.H. BRuneau Calcul d'écoulements incompressibles derrière des obstacles et analyse des solutions transitoires. à paraitre dans les actes du congrès d'Analyse Numérique 1997

[9] P.G. Ciarlet. The Finite Element Method for Elliptic Problems. North-Holland, Amsterdam, New York NY, Oxford, 1978

[10] P.G. Ciarlet. Elasticité tridimensionnelle. Masson, Paris, 1986

[11] P.G. Ciarlet. Plates and Junctions in Elastic Multi-Structures. RMA, Masson, Paris, 1990

[12] P. Destuynder. Une théorie asymptotique des plaques minces en élasticité linéaire. RMA, Masson, 1986 
[13] J. Donea, S. Giuliani, J.P. Halleux. An Arbitrary Lagrangian-Eulerian Finite Element Method for Transient Dynamic Fluid-Structure Interactions. Comp. Methods in Appl. Mech. and Eng. 33, 1982

[14] D. Errate, M. Esteban, Y. Maday. Couplage fluide structure, un modèle simplifié. Note aux C.R.A.S., Paris, t.318, Série I, 1994

[15] R. Glowinski, T-W. Pan, J. Periaux A Fictitious Domain Method for Dirichlet Problem and Applications. Comp. Methods in Appl. Mech. and Eng. 111, 1994

[16] R. Glowinski, T-W. Pan, J. Periaux A Fictitious Domain Method for External Incompressible Viscous Flow Modeled by Navier-Stokes Equations. Comp. Methods in Appl. Mech. and Eng. 112, 1994

[17] C. GRandmont Analyse mathématique et numérique de quelques problèmes d'interactions fluide structure Thèse de doctorat de l'Université Paris VI, 1998

[18] C. Grandmont et Y. Maday. Analysis of a 2D Fluid Structure Interaction. en préparation, 1997

[19] C. Grandmont et Y. Maday. Nonconforming Matching for Incompatible Meshes in Fluid-Structure Interaction. soumis aux actes de la conférence DDM10, Août 1997, Boulder Colorado.

[20] C. Grandmont, V. Guimet et Y. Maday. Numerical Analysis of Some Decouling Techniques for the Approximation of the Unsteady Fluid-Structure Interaction. en préparation, 1997.

[21] L-W. Ho A Legendre Spectral Element Method for Simulation of Incompressible Unsteady Viscous Free-Surface Flow thesis of the Massachusetts Institute of Technology, June 1989

[22] T.J.R. Hughes, W. Liu, T.K. Zimmerman. Lagrangian-Eulerian Finite Element Formulation for Incompressible Viscous Flows. Comp. Methods in Appl. Mech. and Eng. vol 29, 1981

[23] A. Inoue et M. Wakimoto On Existence of Solutions of the Navier-Stokes Equations in a Time Dependent Domain J. Fac. Sci. Univ. Tokyo Sect. IA Math., vol 24, pp $303-319,1977$

[24] M. Lessoine and C. FARHAT Stability Analysis of Dynamic Mesches for Transient Aeroelastic Computatitions 11th AIAA Computational Fluid Dynamics Conference, Orlando, Florida, July 6-9 1993

[25] J.L. Lions Quelques méthodes de résolution des problèmes aux limites non linéaires Dunod, Paris, 1969

[26] H. MoRAnd, R. OHAYON Interaction fluide structure. RMA, Masson, 1992.

[27] B. MAURY . Résolution numérique des équations de Navier-Stokes $2 D$ et $3 D$ avec surface libre par une mèthode Euler-Lagrange arbitraire. Thèse de doctorat de l'Université Paris VI, 1995

[28] J. Mouro et P. LE TALLEC. , Structure en grands déplacement couplées à des fluides en mouvement. Rapport INRIA N²961, 1996

[29] S. Piperno, C. Farhat, B. Larrouturou. Partioned Procedures for the Transient Solution of Coupled Aeroelastic Problems. Part I: Model Problem, Theory, and TwoDimensional application. Comp. Methods in Appl. Mech. and Eng., vol 124, $\mathrm{n}^{\circ} 1-2$, 1995

[30] R. Salvi. On the Existence of a Weak Solution of a Non-Linear Mixed Problem for the Navier-Stokes Equations in a Time Dependent Domain. J. Fac. Sci. Univ. Tokyo Sect. IA Math., vol 32, pp 213-221, 1985 
[31] V.A. Solonnikov. Solvability of a Problem on the Motion of a Viscous Incompressible Fluid Bouded by a Free Surface. Math. USSR Izvestiya, vol $11 \mathrm{~N}^{\circ} 6,1977$

[32] V.A. Solonnikov. On the Transiant Motion of an Isolated Volume of Viscous Incompressible Fluid. Math. USSR Izvestiya, vol 31 No 2,1988 